\title{
MECHATRONIC SYSTEM USED FOR MEASURING THE WEARING MILLING SURFACE OUT
}

\author{
BESNEA, D[aniel] \& SPANU, A[lina] R[odica]
}

\begin{abstract}
The paper concerns with a method for real - time measuring of active surfaces for the devices, during the milling process. The method based on theoretical principle taken from optics, was applied using a mechatronic system actuated by electric stepper motor. The occlusion time of the optical system, which has to scan the object as the speed value is very well known, is the most important physical parameter. The proposed method could be applied for larger device diameter, because of the hexgonal active surface using. In order to measure the time between two switches, we have designed an encoder for the mechatronic system. Its all noise influences could be eliminated during the technological process. The processed signal variation as measuring time function could provide clearly and accurate information about the worn dimension value out.
\end{abstract}

Keywords: Mechatronic systems, laser radiation, accurate measuring, wear milling surface out

\section{INTRODUCTION}

In order to realize a high accuracy dimensional control, the laser radiation could be used. The advantages consist in its main property of spatial and temporal coherence as well as the limited divergence of the fascicle of rays. The laser ray $\mathrm{He}-\mathrm{Ne}$ with $650 \mathrm{~nm}$ emission is used because of the stability of the wavelength. According to the range of dimensional values, the measuring method using the laser radiation could be applied for the value of $1 \mathrm{~mm}$ diameter or greater value, such as $20 \mathrm{~mm}$.

The method principle (Fig. 1) is based on measuring the occlusion time of laser ray, which is moved continuously along a plane. The object has a translational movement or not, depending on the purpose to fulfill.

The laser ray is passing around the object with a rotational speed, as we may infer from Fig. 1. The laser ray movement is done with the translational speed $\mathrm{v}$ that

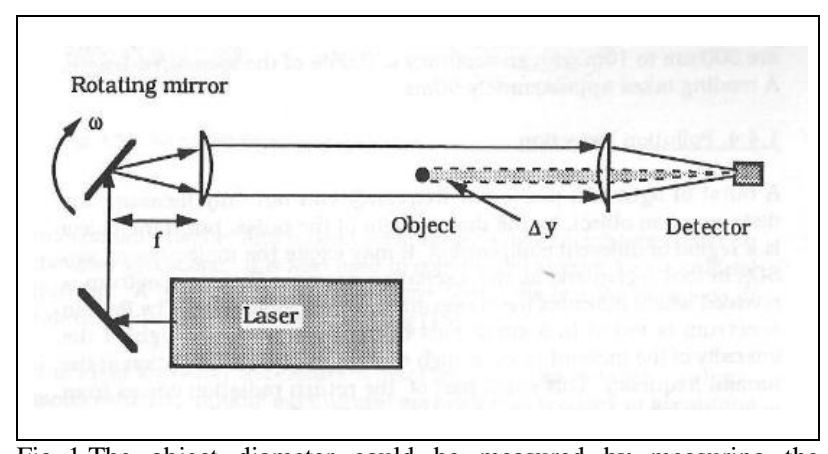

Fig. 1.The object diameter could be measured by measuring the occlusion time of the laser ray [1] depends on the rotational speed of the rotating mirror $\omega$ and could be computed by using the following equation:

$$
v=2 \cdot \omega \cdot f
$$

where $f$ - focal length of the lens.

The object diameter is computed as a function of the $\Delta y$ displacement:

$$
\Delta y=v \cdot \Delta t=2 \cdot \omega \cdot f \cdot \Delta t
$$

Consequently, the reflected ray speed is twice the rotational speed of the mirror, so that the occlusion time value gives directly information about the diameter of the object. The method accuracy depends on the laser ray diameter $\mathrm{d}$, in order to avoid the diffraction. The main requirement regarding the system performances is:

$$
d<<\Delta y
$$

The paper aims to apply this measuring method to devices used for $\mathrm{CNC}$, so that active surface dimensions of the devices having values greater than $1 \mathrm{~mm}$ have to be established. Because of real time measuring, these values are inside an imposed range and the corrections may be applied [2].

\section{THE MECHATRONIC SYSTEM USED FOR MEASURING}

Taking into account all these theoretical principles, we have designed a mechatronic system, which may be used during the technological process, in order to measure the real time for wearing the device out.

We have used an optic focused system with larger sweeping area, so that diameter values for measuring were increased within a known range. The speed value of the ray has been increased too by using a hexagon for reflection (Fig. 2) [2], [3].

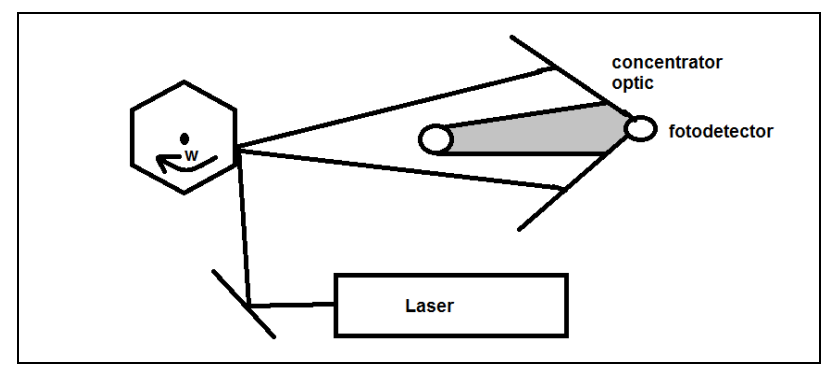

Fig. 2. The principle of the measuring system using the laser radiation 


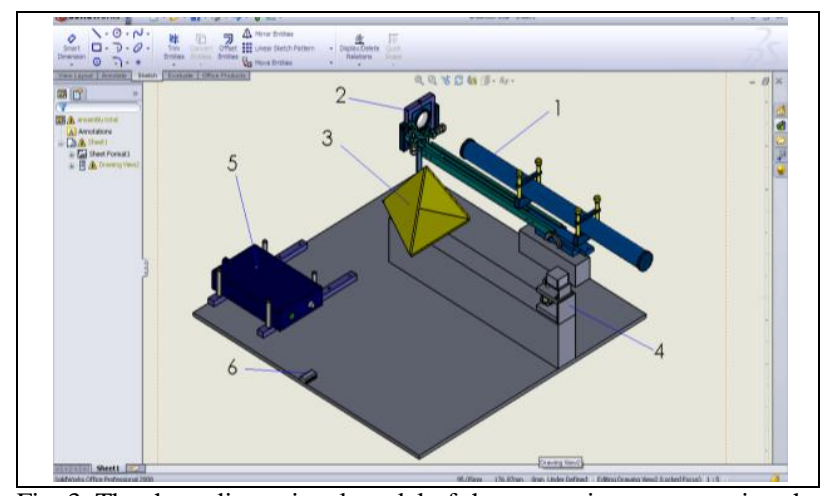

Fig. 3. The three dimensional model of the measuring system using the laser radiation

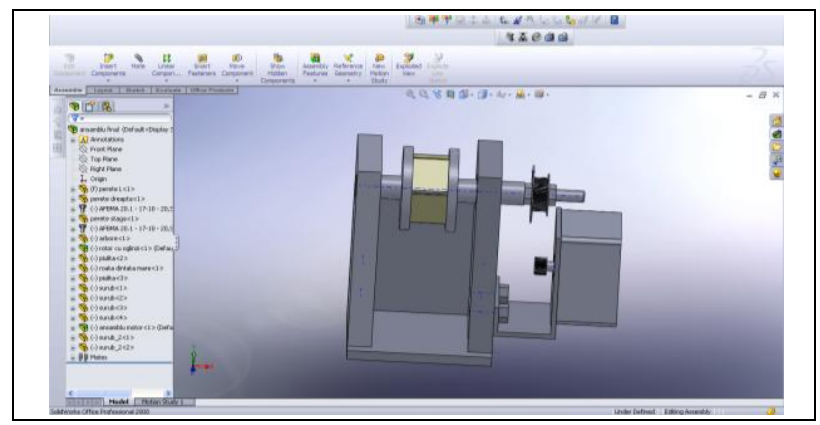

Fig. 4. The sweeping hexagon and the electrical stepper motor

The three dimensional model of the mechatronic system is presented in Fig. 3. We may find the main elements: 1- the He-Ne laser; 2 - the mirror; 3 - the sensor and mirror system; 4 - sweeping system; 5 - laser source; 6 - National Instrument device.

The laser radiation is diverted permanently toward the photo-detection device inside the optic focused system. Only during the object measuring, the laser ray does not touch the photo-detection device and this time value is well known. The main condition that has to be accomplished by the focused optic system is: the laser ray angle of incidence has to be less than $90^{\circ}$ in respect of the perpendicular line to the mirror surface.

The hexagon is actuated by an electric stepper motor (Fig. 4), whose rotational speed may be controlled accurately. We have designed an optical switch by using a hole on the active surface of the rotational mechanism actuating the hexagon, so that we may measure the rotational speed. Meantime, we verify the theoretical results of the speed values. As we know the time between two successive switches, we provide the hexagon rotational speed and we compute the laser ray translational speed too by using (1).

\section{THE CONTROL OF MECHATRONIC SYSTEM}

The electrical stepper motor actuation has been controlled by using LabView software [4], [6] and the National Instruments USB6009 device. We have designed the virtual instruments for controlling the electrical stepper motor, the rotational speed encoder and for the impulse control of the measurement.

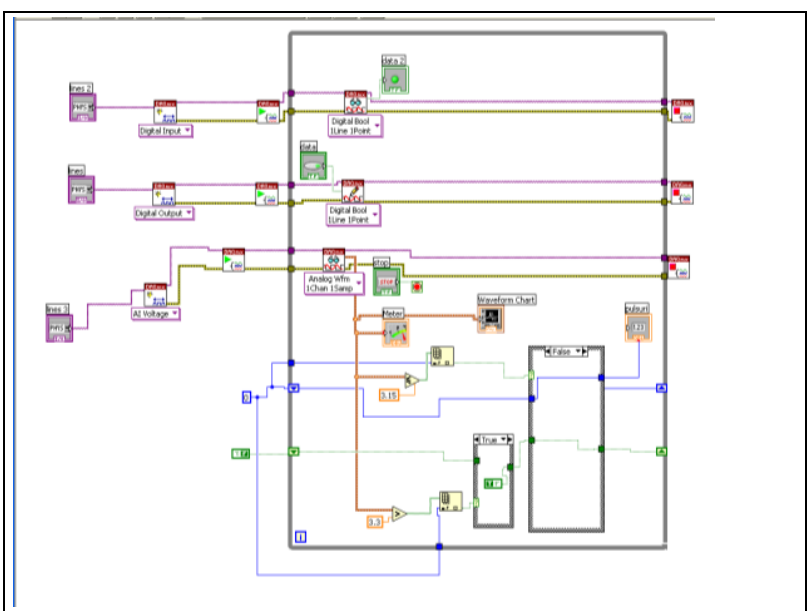

Fig. 5. The block diagram designed for the encoder

As we have mentioned above, the hexagon rotational speed is the same as the electrical motor speed, which is varied as time function by the frequency of phase.

In order to measure the speed, the encoder was designed and the block diagram is presented in Fig. 5. There were designed three virtual channels with digital input and virtual data [5]. The virtual instrument figures out the measured values and a graphic wave has set up the variation as time function. All the impulses are memorized in an array, whose number of elements is the number of impulses. A "while" loop is computed until a logical signal STOP is given from the Front Panel.

Regarding the electric motor control, at first we have to establish the value for the start on frequency $f_{\text {st }}$ as motor couple $\mathrm{M}_{\mathrm{m}}$ and mass inertial constant $\mathrm{J}_{\text {red }}$ function:

$$
f_{s t}=\frac{1}{2 \cdot \pi} \sqrt{\frac{p \cdot M_{m}}{J_{r e d}}}
$$

where $\mathrm{p}$ - the step number of the rotor;

Whether the frequency is in the range [200 steps/s, $300 \mathrm{steps} / \mathrm{s}$ ], the motor couple has the following variation as frequency phase commutation relationship:

$$
\begin{aligned}
& M_{m}=0.1975 e-04 \cdot(f-220)+ \\
& +3.78 e-06 \cdot(f--220) \cdot(f-240)
\end{aligned}
$$

The speed constant value for a certain frequency is accomplished by using the following equations from the mathematical model of the electric stepper motor:

$$
\frac{d \omega}{d t}=\frac{1}{J_{r e d}} \cdot\left(M_{m}-M_{r e z}-D \cdot \omega\right)
$$

where: $\omega$ - the rotational motor speed $[\mathrm{rad} / \mathrm{s}] ; \mathrm{M}_{\mathrm{rez}}-$ the couple which has to be carried out $[\mathrm{Nm}] ; \mathrm{D}$ - the viscous friction constant $[-] ; \mathrm{t}-$ time [s]. We have solved this differential equation as frequency function. Finally, we have found out the optimum frequency for the phase commutation, taking into account the mass inertial constant of the entire moving system $\mathrm{J}_{\text {red }}\left[\mathrm{Kgm}^{2}\right]$.

This method should be applied, if the producer gives the graphic wave of motor couple variation as frequency function. 


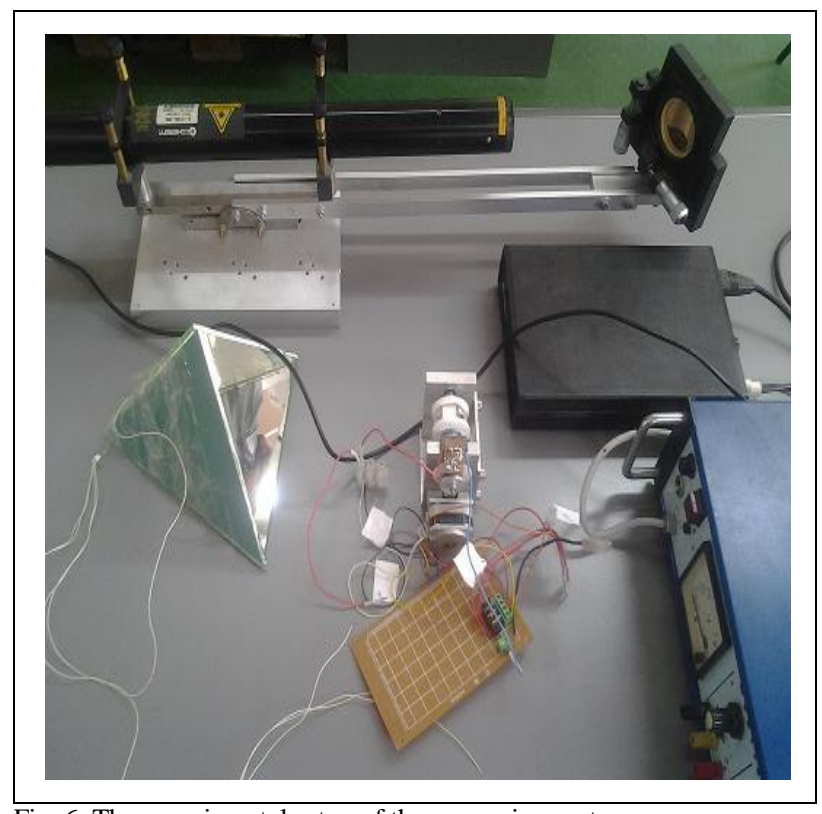

Fig. 6. The experimental setup of the measuring system

\section{THE EXPERIMENTAL SETUP}

We have accomplished the experimental setup of the mechatronic system as Fig. 6 presents.

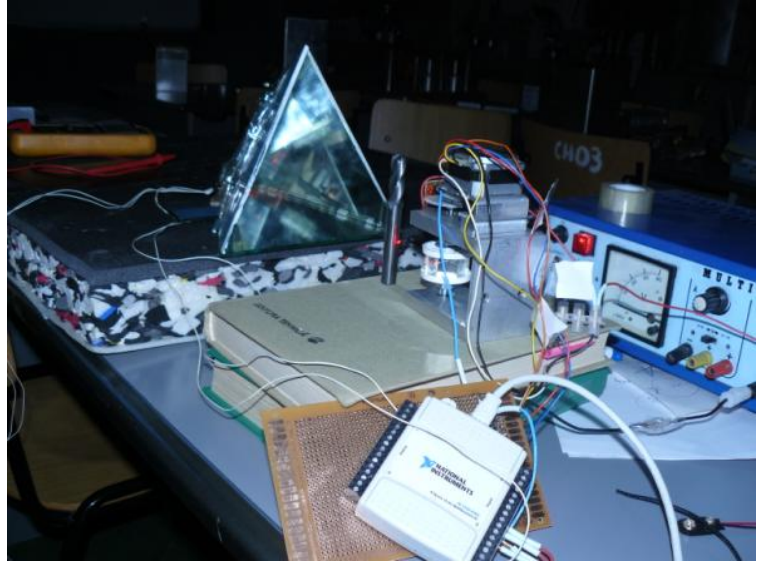

Fig. 7. The milling, the hexagon and the National Instruments USB6009 device

At first the photo-detection device has been calibrated as dependence on the ambient light, in order to provide the presence of laser ray radiation over the electrical photo-resistance surface.

We may apply the proposed method for measuring the wear milling surface out, as we infer from Fig. 7. The milling has the diameter of $15 \mathrm{~mm}$. Fig. 8 presents the main results for the measuring.

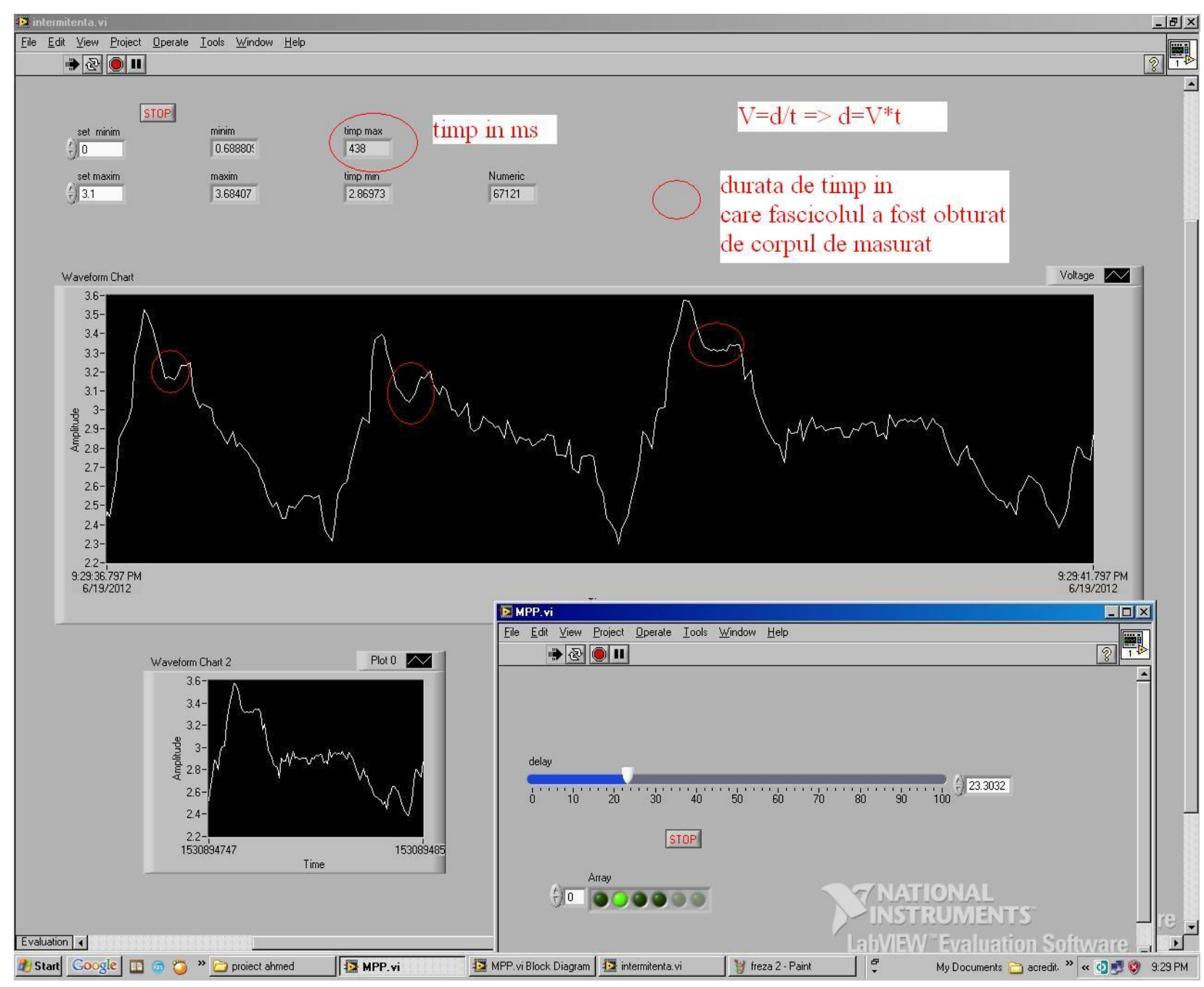

Fig. 8. The measuring results for the $\Phi 15 \mathrm{~mm}$ milling 
The Front Panel shows the values for the minimum measuring time, which is $\mathrm{t}_{1}=2.86973 \mathrm{~ms}$ as well the maximum time value, $\mathrm{t}_{2}=4.38 \mathrm{~ms}$. We have pointed out the period in [ms] when the laser ray is stopped up by the milling, whose dimensions we have to controll. From the graphic wave we infer the variation of milling diameter during the technological process. The wear surface out is measured periodically during the movement.

\section{SIGNAL}
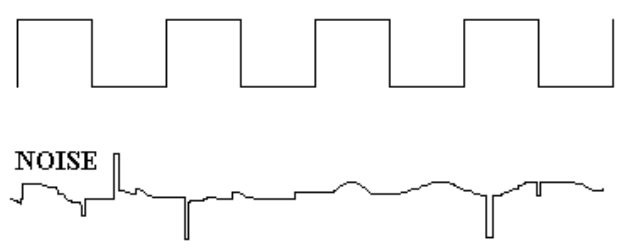

\section{SIGNAL + NOISE}

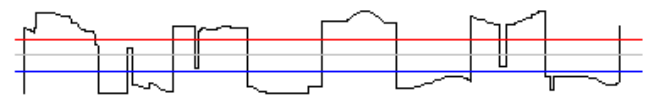

Fig. 9. The noise filter applied during the measurement

Meantime, we may distinguish the period between two phase commutation of the electrical stepper motor, which value is about $23.3032 \mathrm{~ms}$.

Due to electrical noises and variation of light intensity, some bad influences have appeared during the experimental testing. Because of high intensity of laser radiation that is measured as electrical tension variation for the data aquisition analogic input, the expected values were attended. Consequently, following the theoretical principle (Fig. 9), we have applied a noise filter by software computation. The main advantage is the opportunity for modyfing it during the experiment, whether the external influences are dominant. We have proposed it as solution to avoid the electronic amplification, which can not remove the noise.

The experimental results are closely related to the theoretical principles, so that we have found out a qualitative view of wear device out during the technological process. The method could be used as accurate measuring during real time activities.

As future work, we aim to improve the controll system of the electrical motor, as feed-back designing using transfer function of the system and analyzing its dynamic stability.

\section{CONCLUSION}

The authors have develop a theoretical and practical approach to the design of mechatronic system that is useful for real-time dimensional measuring with the laser ray. There were designed virtual instruments using LabView environment for controlling.

The paper concerns with a method for real - time measuring of active surfaces of the devices during the milling process. The method based on theoretical principle taken from optics, was applied using a mechatronic system actuated by electric stepper motor.

The occlusion time of the optical system, which has to scan the object as the speed value is very well known, is the most important physical parameter. The proposed method could be applied for larger device diameter, because of the hexgonal active surface using. In order to measure the time between two switches, we have designed an encoder for the mechatronic system. The laser ray could be used in order to measure the distance, taking into account the flight time of a short impulse.

The control of the electric stepper motor and of the encoder was done by using the LabView development environment. There were designed three virtual instruments: for the electrical stepper motor, for the encoder and for the device which allow the impulse generation. The data acquisition device from National Instruments accomplishes the system.

The processed signal variation as measuring time function could provide clearly and accurate information about the worn dimension value out.

Meantime, we may observe the occlusion time during the measurement. This time value is given by the encoder, so that knowing the period between two switches we may compute the rotational speed of the hexagonal surface.

All the noise influences are eliminated from the final results, by using a filter during the computation. The filter has the band width value variable, as it could be changed during the process, in order to avoid the influence of bad light intensity. It could be an important advantage, when some disturbances happen.

The limitations of proposed method are: the maximum device diameter value is $25 \mathrm{~mm}$; the measurement accuracy is $\pm 0.01 \mathrm{~mm}$; the device axis in front of the scan system should be perpendicular to the sensor axis inside the hexagonal surface.

As future work we aim to improve the dynamic performances of the mechatronic system, especially regarding the encoder and electric stepper motor control. The technical parameters of the control process could be changed, if we use a feed - back system. In such a way, we better control the hexagon rotational speed and the dynamic stability.

\section{REFERENCES}

[1] Steen, W. (1998). Laser Material Processing, Springer-Verlag, ISBN 3-540-76174-8, London

[2] Avarvarei, I.; Dontu, O., Filipoiu, D., Besnea, D., Turcan, O. \& Savastru, D. (2011). Control system, device and technologies for thin wire diameter measurement, Journal of Optoelectronics and Advanced Materials, Vol. 13, No. 8, ISSN 1454-4164, pp. 1030 1036

[3] Popescu, I. (2000). The Physics and Laser Engineering (Fizica si Ingineria Laserilor), Editura Tehnica, ISBN 973-31-1475-8, Bucharest

[4] Munteanu, M. \& Logofatu, B. (2003). Virtual devices (Instrumentatie Virtuala), Editura Credis, ISBN 9773-7701-26-7, Bucharest

[5] Munteanu, M., Logofatu, B. \& Lincke, R. (2003). Virtual devices practical (Aplicatii de Instrumentatie Virtuala), Editura Credis, ISBN 973-7701-27-5, Bucharest

[6] Jelecanin, J., Palm, S. \& Mrcela, T. (2008). Open-loop control and data acquisition of a biomedical research device using LabView development environment, Annals of DAAAM for 2008 \& Proceedings of the $19^{\text {th }}$ International DAAAM Symposium "Intelligent Manufacturing and Automation: Focus on Next Generation of Intelligent Systems and Solutions", 22-25 October, Trnava, Slovakia, ISSN 1726-9679, ISBN 978-3901509-68-1, Katalinic, B. (Ed.), pp. 0679-0680, Vienna 